\title{
Phase error analysis of the LC-Tank CMOS quadrature oscillators
}

\author{
Hossein Miar Naimi ${ }^{a}$ and Hojat Ghonoodi \\ Babol University of Technology, Babol, Iran, P.Box 484 \\ a)h_miare@nit.ac.ir
}

\begin{abstract}
Using an analytical approach, new estimation of phase error in LC-Tank quadrature oscillators is proposed. The mismatches between passive components of LC tanks are considered as the reasons of the phase error. Closed form equations relating LC mismatches to phase errors are presented. To evaluate the analysis, a $5 \mathrm{GHz}$ quadrature LC-Tank oscillator is simulated using TSMC $0.18 \mu \mathrm{m}$ model technology. The results confirm the simplicity and high accuracy of the proposed analysis.
\end{abstract}

Keywords: quadrature oscillator, phase error

Classification: Integrated circuits

\section{References}

[1] B. Razavi, "Design Considerations for Direct-Conversion Receivers," IEEE Trans. Circuits Syst. II, vol. 44, pp. 428-435, 1997.

[2] A. Rofougaran, J. Rael, M. Rofougaran, and A. Abidi, "A 900 MHz CMOS LC-Oscillator with quadrature Outputs," Proc. of the 1996 IEEE Int. Solid-State Circuits Conf., pp. 392-393, 1996.

[3] Y.-H. Hsieh, W.-Y. Hu, S.-M. Lin, C.-L. Chen, W.-K. Li, S.-J. Chen, and D. Chen, "An auto-I/Q calibrated CMOS transceiver for 802.11 g," IEEE J. Solid-State Circuits, vol. 40, pp. 2187-2192, Nov. 2005.

[4] L. Romanò, S. Levantino, A. Bonfanti, C. Samori, and A. L. Lacaita, "Phase Noise And Accuracy In Quadrature Oscillators," IEEE ISCAS, 2004.

[5] I. R. Chamas and S. Raman, "Analysis and Design of a CMOS PhaseTunable Injection-Coupled LC Quadrature VCO (PTIC-QVCO)," IEEE J. Solid-State Circuits, vol. 44, no. 3, March 2009.

[6] D. Leeson, "A simple model of feedback oscillator noise spectrum," Proc. IEEE, vol. 54, no. 2, pp. 329-330, Feb. 1996.

[7] A. Mazzanti and F. Svelto, "A 1.8-GHz Injection-Locked Quadrature CMOS VCO With Low Phase Noise and High Phase Accuracy," IEEE Trans. Circuits Syst., vol. 53, no. 3, March 2006.

\section{Introduction}

One of the most significant sections of many communication systems are oscillators with quadrature outputs [1]. There are different ways to generate 
quadrature outputs. A straightforward way is using a four-stage differential ring oscillator, but a more common with higher performance topology is two coupled LC-Tank CMOS oscillators. Different ways have been proposed to couple two CMOS LC-Tank oscillators. The first method is parallel coupling proposed by A. Rofougaran [2]. In this topology two additional transistors are added in parallel to one transistor of the oscillator. Using the gates of the parallel transistors, the oscillators are coupled as shown in Fig. 1.

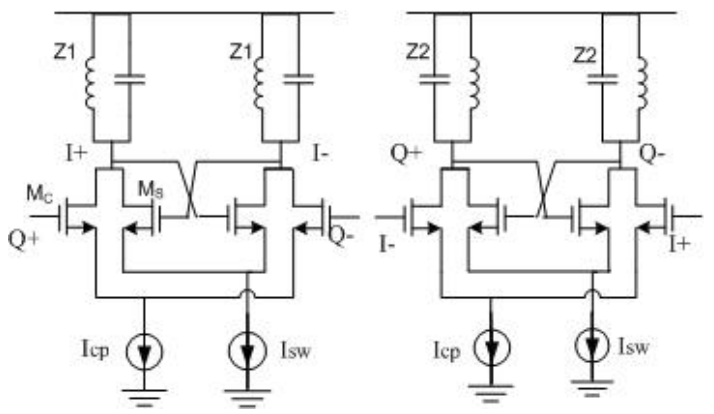

Fig. 1. the parallel coupled quadrature oscillator

In fully match parts, applying Barkhausen's phase criterions implies the 90 degrees phase difference between outputs. Mismatches, like device mismatch, layout asymmetries, and parasitic effects cause phase error. The phase error affects the overall performance of the systems incorporating the quadrature oscillators [3]. This shows the significance of accuracy of the outputs' relative phase. Some references investigate the phase noise of the above oscillators and some other study the phase error using simulation. This tradeoff has been mentioned in several papers $[4,5]$. Having simple accurate equations makes the optimal design straightforward. In this paper closed form equations relating phase error and mismatches are proposed. The rest of the paper is as follows: In section 2, for ideal case and without any mismatches, the quadrature oscillator is analyzed and some attractive aspect of the oscillator is explained. In section 3 the effect of mismatches on phase accuracy is investigated. Simulation results and comparisons are introduced in section 4.

\section{LC-Tank quadrature oscillator}

LC-Tank quadrature oscillators have the topology shown in Fig. 1. In this figure using coupling transistors, shown as $\mathrm{M}_{\mathrm{C}}$, the outputs of each oscillator are fed to the inputs of another. A simple analysis is performed under fully matched devices and components. In oscillation under conditions mentioned above, we can write equations 1 - 2 for four voltages shown in figure 1 .

$$
\begin{gathered}
V_{I \pm}=\mp A \cos (\omega t) \\
V_{Q \mp}=\mp A \cos (\omega t+\theta)
\end{gathered}
$$

In above equations, each block generates differential outputs so 180 degrees phase difference is considered for single ended outputs of each block. Because 
of differential inputs each block experiences, the source of transistor have a constant voltage equivalent to a small signal ground. So the current of each transistor can be written as $I=G m . V$ where $\mathrm{Gm}$ is the "transconductance" of each transistor. In node $V_{Q+}$ we write the following simple equation.

$$
-V_{Q^{+}}=\left(I_{I^{-}}+I_{Q^{-}}\right) \cdot Z
$$

In above equation $\mathrm{Z}$ is the impedance of LC Tank at oscillation frequency. Writing equation (3) in term of phasors leaves to equation (4).

$$
-e^{j \theta}=\left(G_{m c} e^{j 0}-G_{m s} e^{j \theta}\right) \cdot Z
$$

In (4) $G_{m c}$ and $G_{m s}$ are the transconductance of coupling and switching transistors of the oscillator. Equation (4) can be simply expressed as (5).

$$
e^{j \theta}=\frac{G_{m c} \cdot Z}{G_{m s} \cdot Z-1}
$$

We can write similar equation for node $V_{I^{+}}$as shown in (6) that is reduced to $(8)$.

$$
\begin{gathered}
-V_{I^{+}}=\left(I_{Q^{+}}+I_{I^{-}}\right) \cdot Z \\
-e^{j \pi}=\left(G_{m c} e^{j \theta}+G_{m s} e^{j 0}\right) \cdot Z \\
e^{j \theta}=-\frac{G_{m s} \cdot Z-1}{G_{m c} \cdot Z}
\end{gathered}
$$

Multiplying (5) and (8) concludes the following significant equation, proving the quadrature nature of the single ended outputs. One can simply show that two differential output are also quadrature.

$$
e^{j 2 \theta}=-1 \Rightarrow \theta=\pi / 2
$$

\section{Phase error caused by mismatch between LC-tanks}

The above calculations were performed when two LC-Tanks have no mismatches. Under such a condition the phase difference between outputs was 90 degrees but simulations show that when two LC tanks are not exactly the same the phase difference between outputs deviates from 90 degrees. In this section the phase error is related to mismatches of LC tanks and a closed form equation is extracted. To perform this we suppose impedances $Z_{1}$ and $\mathrm{Z}_{2}$ for two tanks as shown in figure 1 .

For a general solution we assume below expressions for four single ended outputs.

$$
\begin{gathered}
V_{I^{-}}=A \cos (\omega t) \\
V_{Q^{+}}=A \cos \left(\omega t+\theta_{1}\right) \\
V_{I^{+}}=A \cos \left(\omega t+\theta_{2}\right) \\
V_{Q^{-}}=A \cos \left(\omega t+\theta_{3}\right)
\end{gathered}
$$

The current voltage relation for $V_{I^{+}}$is as (14)

$$
-V_{I^{+}}=\left(I_{Q^{+}}+I_{I^{-}}\right) \cdot Z_{1}
$$




$$
\begin{gathered}
I_{I^{-}}=G_{m s} A \cdot \cos (\omega t), I_{Q^{+}}=G_{m c} A \cdot \cos \left(\omega t+\theta_{1}\right) \\
I_{Q^{+}}+I_{I^{-}}=G_{m s} A\left(\cos \omega t+k \cos \left(\omega t+\theta_{1}\right)\right), k=\frac{G_{m c}}{G_{m s}}
\end{gathered}
$$

The current of (16) passes through $\mathrm{Z}_{1}$ and builds up $V_{I+}$. The first term of (16) has a real phasor of size 1 and angle of zero. The second term has a complex phasor of size $\mathrm{k}$ and angle of $\theta_{1}$. Looking at Fig. 2 one can simply find that the phase of (16) is as (17).

$$
\angle\left(I_{Q^{+}}+I_{I^{-}}\right)=\frac{\theta_{1}}{2}+\alpha
$$

As we know without any mismatch the phase error is zero and $\theta_{1}=90$. Mismatches causes $\theta_{1}$ deviates from 90 degrees but it is close to 90 yet. To compute $\alpha$ we use the approximation of $\theta_{1} \approx 90$ in following calculations.

In figure 2 (a) we can write

$$
\frac{\sin y}{\sqrt{2}}=\frac{\sin \alpha}{k-1} \Rightarrow \sin \alpha=\frac{(k-1)}{\sqrt{2}} \frac{\tan y}{\sqrt{1+\tan ^{2} y}}
$$

Using $\theta_{1} \approx 90$ approximation we will have

$$
\tan y=\frac{1}{k} \Rightarrow \sin \alpha=\frac{k-1}{\sqrt{2\left(k^{2}+1\right)}} \Rightarrow \alpha=\sin ^{-1}\left(\frac{k-1}{\sqrt{2\left(k^{2}+1\right)}}\right)
$$

So the phase of $(16)$ is as

$$
\angle\left(I_{Q^{+}}+I_{I^{-}}\right)=\frac{\theta_{1}}{2}+\alpha
$$

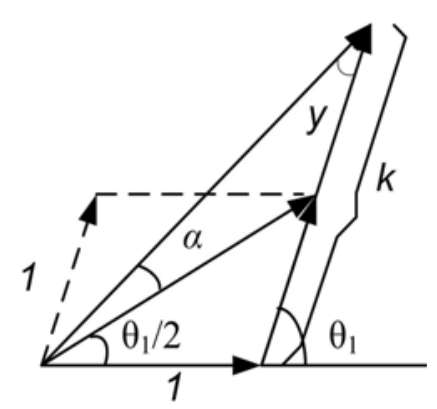

(a)

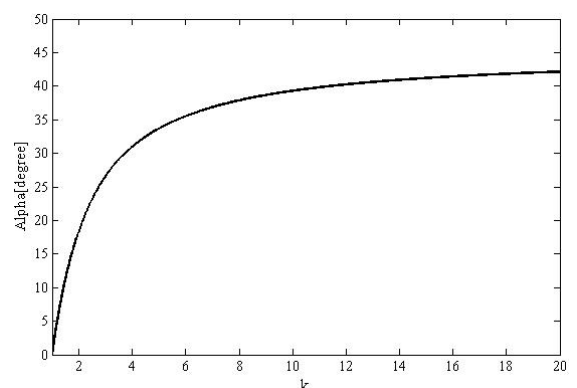

(b)

Fig. 2. (a) phasor diagram of (16). (b) the dependency between $k$ and $\alpha$

So considering (14), the relation of (20) holds for $\theta_{1}, \theta_{2}$.

$$
\theta_{2}=\frac{\theta_{1}}{2}+\alpha+\varphi_{1}+\pi \quad, \quad \varphi_{1}=\angle Z_{1}
$$

Using similar approach for $V_{Q^{+}}, V_{I^{-}}$and $V_{Q^{-}}$we find following relations among different angles.

$$
V_{Q^{+}}: \quad \theta_{1}=\frac{\theta_{3}}{2}+\alpha+\varphi_{2} \quad, \quad \varphi_{2}=\angle Z_{2}
$$




$$
\begin{gathered}
V_{I^{-}}: \quad \pi=\frac{\theta_{2}}{2}+\frac{\theta_{3}}{2}+\alpha+\varphi_{1} \\
V_{Q^{-}}: \quad \theta_{3}=\frac{\theta_{1}}{2}+\frac{\theta_{2}}{2}+\alpha+\varphi_{2}+\pi
\end{gathered}
$$

Angle of impedance of tanks at oscillation frequency is as (24) and (25).

$$
\begin{aligned}
& \varphi_{1}=\tan ^{-1}\left[\frac{R_{p 1}\left(1-L_{1} C_{1} \omega^{2}\right)}{L_{1} \omega}\right] \\
& \varphi_{2}=\tan ^{-1}\left[\frac{R_{p 2}\left(1-L_{2} C_{2} \omega^{2}\right)}{L_{2} \omega}\right]
\end{aligned}
$$

Here $R_{p 1,2}$ are equivalent parallel resistors in the LC tank. Equations (20) to (25) form a six unknown equations system. Simple investigations show that we have $\theta_{2}=\pi$ and $\theta_{3}=\theta_{1}+\pi$. Indeed under unbalance conditions, the single ended output save their differential nature. Applying this, (20) is reduced to $(26)$.

$$
\theta_{1}=-2 \varphi_{1}-2 \alpha
$$

To continue, assume that the difference of angles of two impedances be $\Delta \varphi$ as defined in (27).

$$
\Delta \varphi=\varphi_{2}-\varphi_{1}
$$

Summing (20) to (23), leaves equation (28).

$$
\varphi_{1}+\varphi_{2}=-\frac{\pi}{2}-2 \alpha
$$

From (27) and (28) we can express $\varphi_{1}, \varphi_{2}$ as (29) and (30).

$$
\begin{aligned}
& \varphi_{1}=-\frac{\pi}{4}-\alpha-\frac{\Delta \varphi}{2} \\
& \varphi_{2}=-\frac{\pi}{4}-\alpha+\frac{\Delta \varphi}{2}
\end{aligned}
$$

From (29) and (30) equation (26) can be written as (31).

$$
\theta_{1}=-2 \varphi_{1}-2 \alpha=\frac{\pi}{2}+\Delta \varphi
$$

Equation (31) shows that the phase error under mismatch is the difference between angles of two impedances. Substituting $\varphi_{1}, \varphi_{2}$ from (29) and (30) in (24) and (25) leads to (32) and (33).

$$
\begin{aligned}
& \tan \left(\frac{\pi}{4}+\alpha+\frac{\Delta \varphi}{2}\right)=-\frac{R_{p 1}\left(1-L_{1} C_{1} \omega^{2}\right)}{L_{1} \omega} \\
& \tan \left(\frac{\pi}{4}+\alpha-\frac{\Delta \varphi}{2}\right)=-\frac{R_{p 2}\left(1-L_{2} C_{2} \omega^{2}\right)}{L_{2} \omega}
\end{aligned}
$$

$\Delta \varphi$ and $\omega$ are the solutions of equations system of (32) and (33). The direct solution of the system is very complicated. So we introduce an approximate method. We assume $\beta_{1}=\alpha+\Delta \varphi / 2$ and $\beta_{2}=\alpha-\Delta \varphi / 2$. We can write,

$$
\tan \left(\beta_{1,2}+\frac{\pi}{4}\right)=\frac{\sin \left(\beta_{1,2}+\frac{\pi}{4}\right)}{\cos \left(\beta_{1,2}+\frac{\pi}{4}\right)}=\frac{\sin \left(\beta_{1,2}\right)+\cos \left(\beta_{1,2}\right)}{\cos \left(\beta_{1,2}\right)-\sin \left(\beta_{1,2}\right)}
$$


$\beta_{1,2}$ have generally small values, so it is reasonable to write $\cos (\beta)=1$ and $\sin (\beta)=\beta$, so from (34) we will have following equation.

$$
\tan \left(\beta_{1,2}+\frac{\pi}{4}\right)=\frac{1+\beta_{1,2}}{1-\beta_{1,2}}
$$

Using above approximation (35) is simplified to equation (36)

$$
\frac{1+\beta_{1,2}}{1-\beta_{1,2}}=-\frac{R_{p 1,2}\left(1-L_{1,2} C_{1,2} \omega^{2}\right)}{L_{1,2} \omega}
$$

From (36) can be written as (37).

$$
\frac{1+\beta_{1}}{1-\beta_{1}}-\frac{1+\beta_{2}}{1-\beta_{2}}=\frac{R_{p 2}\left(1-L_{2} C_{2} \omega^{2}\right)}{L_{2} \omega}-\frac{R_{p 1}\left(1-L_{1} C_{1} \omega^{2}\right)}{L_{1} \omega}
$$

Substituting $\beta_{1}=\alpha+\Delta \varphi / 2$ and $\beta_{2}=\alpha-\Delta \varphi / 2$, the left hand of (37) can be rewritten as (38)

$$
\begin{aligned}
\frac{1+\beta_{1}}{1-\beta_{1}}-\frac{1+\beta_{2}}{1-\beta_{2}} & =\frac{2 \beta_{1}-2 \beta_{2}}{1-\beta_{1}-\beta_{2}+\beta_{1} \beta_{2}} \\
& =\frac{2 \Delta \varphi}{1-2 \alpha+\alpha^{2}-\frac{\Delta \varphi^{2}}{4}} \cong \frac{2 \Delta \varphi}{1-2 \alpha+\alpha^{2}}=\frac{2 \Delta \varphi}{(1-\alpha)^{2}}
\end{aligned}
$$

Ignoring $\Delta \varphi^{2}$ (37) is reduced to (39).

$$
\frac{2 \Delta \varphi}{(1-\alpha)^{2}}=\frac{R_{p 2}\left(1-L_{2} C_{2} \omega^{2}\right)}{L_{2} \omega}-\frac{R_{p 1}\left(1-L_{1} C_{1} \omega^{2}\right)}{L_{1} \omega}
$$

(39) is the relation between phase error and components of two LC tanks. Assuming the same value for the resistance of two tanks as $R_{p}$, (39) can be written as (40).

$$
\begin{aligned}
\Delta \varphi & =\frac{R_{p}(1-\alpha)^{2}}{2 \omega}\left[\frac{\left(1-L_{2} C_{2} \omega^{2}\right)}{L_{2}}-\frac{\left(1-L_{1} C_{1} \omega^{2}\right)}{L_{1}}\right] \\
& =\frac{R_{p}(1-\alpha)^{2}}{2 \omega}\left(\frac{\Delta L}{L_{1} L_{2}}+\Delta C \omega^{2}\right)
\end{aligned}
$$

Where $\Delta C=C_{1}-C_{2}$ and $\Delta L=L_{1}-L_{2}$. Using quality factor of tanks, above expression can be written in a better form as follows:

$$
Q=R_{p} \sqrt{\frac{C}{L}} \Rightarrow R_{p}=Q L \omega_{0}=\frac{Q}{C \omega_{0}}
$$

Replacing $R_{p}$ from (41) in (40) the phase error is represented as (42)

$$
\Delta \varphi=\frac{Q}{2}\left(\frac{\omega_{0}}{\omega_{\text {osc }}} \frac{\Delta L}{L}+\frac{\omega_{o s c}}{\omega_{0}} \frac{\Delta C}{C}\right)(1-\alpha)^{2}
$$

We suppose $\omega_{\text {osc }} \cong \omega_{0}$ that concludes the below simplified expression for phase error.

$$
\Delta \varphi=\frac{Q}{2}\left(\frac{\Delta L}{L}+\frac{\Delta C}{C}\right)(1-\alpha)^{2}
$$

As (43) shows, decreasing $\mathrm{Q}$ and increasing $\alpha$ causes phase error be less sensitive to mismatches in LC tanks. On the other hand phase noise is 
inversely proportional to $\mathrm{Q}$, where $L(\Delta \omega) \propto 1 / Q^{2}$ and directly proportional to coupling factor $(\mathrm{k})$, so there is a tradeoff between phase noise and phase error $[6,7]$. (Notice to Fig. 2 (b)).

As mentioned, decreasing phase error by changing $\mathrm{Q}$ or the relative ratio of coupling transistor and main transistor results in increasing phase noise. As we know, phase error is equal to the phase difference of two LC tank impedances. If we decrease phase difference of LC tanks' impedance without any change in the $\mathrm{Q}$ or coupling factor circuit, phase error will decrease without any increasing in phase noise. From (39), adding a small! Controlled parallel resistor to one of $\mathrm{LC}$ tanks changes $\mathrm{R}_{\mathrm{p}}$ and consequently decreases phase error.

\section{Simulation results}

To evaluate the proposed analytical results, a $5 \mathrm{GHz}$ oscillator is designed and simulated using $0.18 \mu \mathrm{m}$ CMOS TSMC model and different experiments have been performed. The LC tank comprises of a $2-\mathrm{nH}$ inductor and 0.5-pF capacitor. Transistors $\mathrm{M}_{\mathrm{C}}$ and $\mathrm{M}_{\mathrm{S}}$ have the same aspect ratio [W/L] of $10[\mu \mathrm{m}] / 0.18[\mu \mathrm{m}]$. To verify the dependency of phase error to the oscillator coupling factor, the current of main transistors is kept constant at $1 \mathrm{~mA}$, while the current of coupling transistors is varied from $1 \mathrm{~mA}$ to $2.5 \mathrm{~mA}$. Dotted

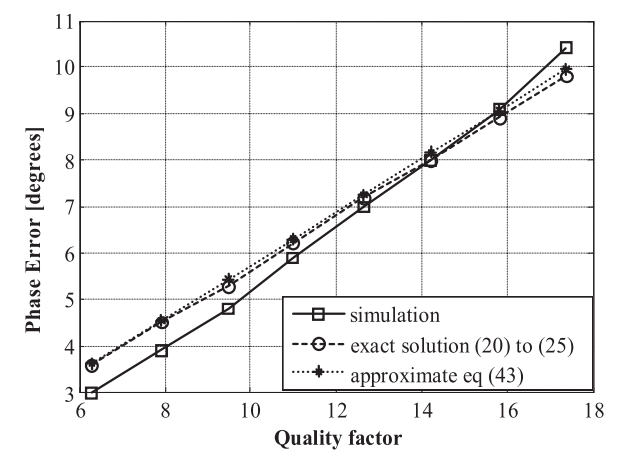

(a)

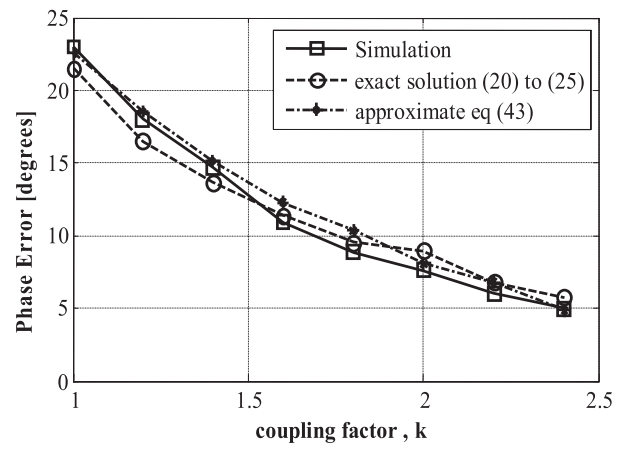

(b)

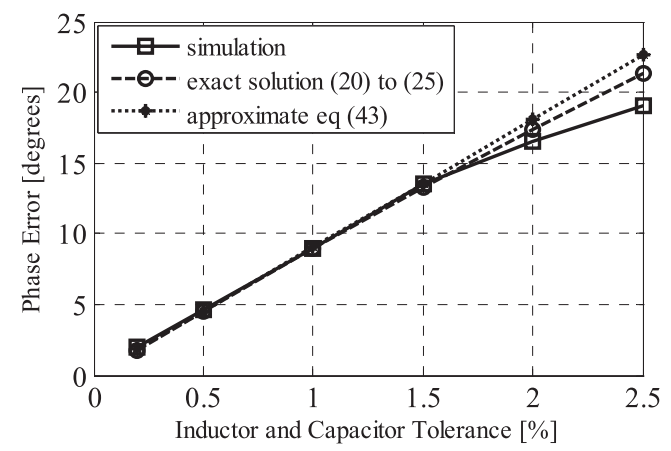

(c)

Fig. 3. (a) Phase error versus $\mathrm{Q} ; \mathrm{k}=1$ and $1 \%$ mismatches in inductors and capacitors. (b) Phase error versus $\mathrm{k}$; $5 \%$ mismatches in inductors. (c) phase error versus mismatches in inductors and capacitors; $\mathrm{k}=1$ 
curves in Fig. 3 (a) shows the simulated phase errors versus quality factor for a mismatch of $1 \%$ on tank capacitance and inductance compared with result of equations (20) to (25) and approximate equation (43). Fig. 3 (b) shows the phase error versus coupling factor for $5 \%$ mismatches in inductors with a $\mathrm{Q}$ of 10. Fig. 3 (c) shows phase error versus mismatches in inductors and capacitors.

As one can see the proposed analysis exactly meet the simulation results.

\section{Conclusions}

A new approach to analysis the phase error of the parallel coupled quadrature LC tank oscillators has been proposed and new closed form equation was introduced. All behavioral aspects seen in simulations have been estimated by the proposed model. For more study we are focusing to design a self phase error compensated quadrature oscillator. 